\title{
La voz del alumnado: Una investigación narrativa acerca de lo que siente, piensa, dice y hace el alumnado de Magisterio de Educación Física en su formación inicial.
}

\author{
Berenice Mattos Medina* \\ Esther Prados Megías ** \\ Daniela Padua Arcos ${ }^{* *}$
}

\begin{abstract}
RESUMEN: Esta investigación se orienta hacia a la descripción e interpretación acerca de lo que siente, piensa, dice y hace el alumnado de magisterio de educación infantil en su formación inicial. Para llevar a cabo esta investigación he combinado distintos instrumentos de recogida de informaciones: observación participante, grupos de discusión, diario de campo, entrevistas individuales y documentos del alumnado. Propongo que se continúe investigando para conducir a la formación hacia la posibilidad de vivir la complejidad, la incertidumbre y la tensión de la vida real en contextos educativos naturales y que se amplíe la reflexión de la formación inicial.
\end{abstract}

Palabras claves: estudiantes. Educación. Antropología cultural

\section{El COMIENZO...MOTIVACIONES Y DECISIONES}

Toda investigación o proyecto se inicia con un conjunto de consideraciones acerca de aquella parte del mundo social que se desea estudiar y, que en algunos casos, se trata de cuestiones muy cercanas a nuestras prácticas. Experiencias, lecturas y reflexiones sobre el tema son guiadas por un conjunto de preguntas acerca de la naturaleza de lo que se desea investigar y la manera de abordarlo, tomando en cuenta lo que otros hicieron antes que nosotros, mirando al mundo y preguntándose acerca de las posibilidades y los medios para acceder a él. Ingresé en el programa de Doctorado en la

\footnotetext{
'Professora. Instituto de Educação. Fundação Universidade Federal do Rio Grande (FURG). Rio Grande. RS, Brasil. E-mail: berecaprof@hotmail.com

"Professora titular do Departamento de Educação da Universidade de Almería - Espanha

"'Professora titular do Departamento de Didática e Organização Escolar da Universidade de Almería - Espanha
} 
Universidad de Almería con la inquietud de comprender la formación inicial desde la óptica del alumnado. Comencé a cuestionarme las aportaciones que la formación inicial iba dejando en su persona, en sus vidas personales y profesionales. Aspiraba acercarme en cierta medida a la persona, a las actitudes, acciones, sentimientos, vivencias del alumnado, para que así, conociendo, indagando, reflexionando... pudiese mejorar mi docencia a partir de la comprensión de sus experiencias.

A partir de las reflexiones que me iban surgiendo me he planteado que escuchar al alumnado sería una forma de vislumbrar el proceso formativo desde la visón que ellos y ellas tenían. Oír sus aportaciones, sensaciones, sentimientos, experiencias, dificultades, pensamientos, girar la mirada hacia lo que ellos y ellas tenían a decir acerca de la formación inicial sería la perspectiva que orientaría este proceso de investigación. Podría aportar otra forma de mirar y comprender el proceso formativo desde sus puntos de vista y que el alumnado podría tomar conciencia de la importancia de reflexionar desde su práctica, ya que exponer de forma explícita sus intereses, motivaciones, historias, experiencias...desvelaría elementos sobre los que construyen su formación. Así que, tras ordenar y estructurar las cuestiones planteadas llegamos a la formulación que orientaría este trabajo, la descripción e interpretación acerca de lo que siente, piensa, dice y hace el alumnado de magisterio de educación infantil en su formación inicial. Una investigación que trata de aportar los testimonios en primera persona de los y las participantes que forman parte de ella, dónde tratamos de comprender las creencias, sentimientos, conflictos, aprendizajes... que dan "cuerpo" a su futura formación. A esta investigación le interesaba el alumnado de tercero por estar en el final de la formación y por el último año estar vinculado a la experiencia de las prácticas docentes en la escuela (practicum).

\section{Aproximaciones ePISTEMOLÓgICAS QUE fUNDAMENTAN EL ENTRAMADO DE LO CORPORAL EN LA FORMACIÓN INICIAL}

En la actualidad nos vemos obligados a enseñar de un modo distinto a cómo aprendimos como alumnos y alumnas. La exigencia 
de educar a éstos en una situación de cambio como el que se produce en la sociedad post-moderna se convierte en un reto difícil de afrontar. El profesorado necesita aprender nuevos contenidos y metodologías, nuevas estrategias didácticas, así como incorporar nuevas actitudes, creencias y habilidades docentes con las que poder comprender el tipo de cultura con la que acceden alumnos y alumnas a la escuela y discernir las nuevas referencias en torno a las cuales el mundo de la infancia y adolescencia va construyendo su identidad personal.

El debate sobre educación suele girar siempre sobre los medios para llevarla a cabo y pocas veces sobre sus fines. Existe más preocupación por el cómo hacer que por el para qué hacer, cuestión que es mucho más evidente en la educación universitaria. Si en los primeros niveles educativos se estuviese de acuerdo en admitir, mejor asumir, que la educación ha de servir para aprender a ser más que para preparar el acceso a los estudios superiores y/o al mundo laboral, en la universidad, se haría más evidente la influencia de otras teorías y podríamos preguntar de forma más abierta, tanto al alumnado como al profesorado, ¿para qué vienen a la universidad? Tal vez, muchas personas dudarían de la respuesta, pero seguro, que pocas contestarían que para formarse como personas, como ciudadanos (SAVATER, 2003).

La simple acumulación de contenidos teóricos, de todas y cada una de las disciplinas que integran el currículo de la formación inicial, no garantizan el desarrollo de capacidades, actitudes y comportamientos que se requieren para una eficaz intervención educativa. El papel de la formación inicial (GONZÁLEZ; ROMERO, 2004) no debe centrarse tanto, en desarrollar pequeñas parcelas de contenidos de las asignaturas y áreas que conforman el currículo formativo, como en diseñar el perfil profesional y humano que demanda la sociedad y la escuela. Según esta autora, hoy por hoy, se demanda al docente en general, y en particular en el área de Educación Física, que conozcan y pueda abordar situaciones de prácticas educativas. De ahí la necesidad de afrontar el proceso de formación inicial desde una perspectiva integral y de desarrollo humano y no tanto desde una perspectiva técnica y/o didáctica. 
Es necesario, pues, reflexionar acerca del papel de la educación en el proceso de formación, ya que se siguen manteniendo modos, estructuras y conocimientos muy tradicionales, en escasa consonancia con las demandas para una nueva sociedad y persona. La educación necesita despertar la curiosidad en el alumnado y transmitir unos conocimientos adaptados a la civilización del futuro, que ayuden a éste a interpretar el mundo en que vive y contribuir al desarrollo de sus conocimientos, habilidades y actitudes para hacer que la sociedad evolucione. Preparar al alumnado para actuar en un mundo postindustrial caracterizado por la flexibilidad, la adaptabilidad, la creatividad, el aprovechamiento de las oportunidades, la colaboración, el perfeccionamiento continuo y la resolución de problemas para desenvolverse en la sociedad del riesgo y de la incertidumbre (BECK, 1997).

Uno de los mayores retos ante este panorama de incertidumbre y cambio puede ser la propuesta de una pedagogía que se fundamente en la flexibilidad, creatividad y dialogicidad. Una pedagogía que sea flexible para acoger las necesidades del alumnado así como las de la sociedad, que supere la verticalidad de los currículos rígidos, que sea creativa para que el estudiante deje de ser pasivo, superando la receptividad, la repetición y la acomodación, y finalmente que sea dialógica dado que, como apunta Freire (2002), el conocimiento se obtiene a través del diálogo.

Fraile et al., (2004) plantea la necesidad de generar un cambio substancial en el concepto de ciencia y de educación con nuevos modelos de intervención educativa. Las corrientes socio-críticas fomentan nuevos hábitos y costumbres entre los ciudadanos recuperando el valor de las emociones y de la identidad individual. Este cambio suscita el replanteamiento (BOLÍVAR, 1995) de la formación del profesorado debiendo analizar las prácticas y revisar desde la coherencia las actuaciones, tanto en el ámbito genérico de la educación, como en el específico de lo corporal. Por lo tanto, no podemos limitarnos a enseñar conocimientos sino educar para la vida. La educación en valores representa recuperar para la escuela su función educativa y no sólo reproducir aquellos valores y actitudes socialmente vigentes. 


\section{Construyendo el PROCeso metodológico de LA INVEStigación DENTRO DEL PARADIGMA CUALITATIVO}

A lo largo del proceso de investigación hemos tomado algunas decisiones que han ido guiando este trabajo como por ejemplo situar esta investigación dentro del paradigma cualitativo. Un modelo de investigación que se interesa por las diferencias, por la individualidad y por las características de cada persona lo que nos ha permitido comprender los significados que las personas construyen de su propio contexto. Una investigación dónde los alumnos/as son considerados como protagonistas de la investigación y, para descubrir, entender y describir el significado de una experiencia vivida desde la perspectiva de los protagonistas.

El modelo interpretativo es el que más nos ha interesado en este trabajo de investigación, ya que se preocupa en indagar cómo los distintos actores construyen y reconstruyen la realidad social mediante la interacción con los restantes miembros de su comunidad. En este sentido se hace indispensable tener en cuenta la interpretación que ellos mismos realizan de los porqués y para qué de sus acciones y de la situación en general (ANGUS, 1986; ERICKSON, 1986; SMITH, 1987 citado en GOETZ; LECOMPTE, 1998). Como las cosas no siempre son lo que parecen ser, para entender los significados encubiertos o implícitos en una situación o contexto, es importante no quedarse en la superficie, en lo que aparentemente se observa, sino bucear y buscar en lo más profundo, tal y como apunta Geertz (1973 citado en FLICK, 2004, p.33), para buscar "descripciones gruesas" o interpretación.

En esta investigación hemos optado por un estilo narrativo, ya que permite analizar el discurso desde una perspectiva más real, o lo que a mí me gusta llamar un discurso que va unido a la vida, a las vivencias y a la experiencia de los y las participantes. Las experiencias de la investigación se tienen que transformar en textos y comprenderse a partir de éstos. Un proceso de investigación tiene hallazgos en la medida en que éstos se puedan encontrar en informes, 
sin importar quiénes estuvieron implicados en la investigación y qué experiencias tuvieron. Al respecto Flick (2004, p. 254) dice que:

La escritura es relevante en investigación cualitativa cuando tiene estos tres aspectos: presenta los hallazgos de un proyecto; cuando se pueda considerar como punto de partida para evaluar las actuaciones que conducen a ellos, y finalmente, como punto de salida para las consideraciones reflexivas sobe el estado global de la investigación en conjunto

Como señala Clandinin y Connelly (1995), la importancia de usar la narrativa en la investigación educativa es que los humanos somos personas narradoras, las cuales individualmente y socialmente, somos portadoras de vidas narradas. La narrativa permite también arropar con más naturalidad la expresión de los pensamientos, de los sentimientos, de los deseos de las personas implicadas en la investigación. Es por ello que el informe narrativo también forma parte del proceso de investigación etnográfica. Para reflexionar acerca de lo que aporta las personas investigadas, el estilo narrativo permite analizar el discurso desde una perspectiva más original, desde la óptica de que es un discurso unido a la vida, a las vivencias y a la experiencia.

\section{LA VARIABILIDAD INSTRUMENTAL: UN RECORRIDO NARRADO DEL USO DE LOS INSTRUMENTOS DE RECOGIDA DE INFORMACIÓN}

Una de las cuestiones importantes en investigación etnográfica es lo relativo al significado de la experiencia vivida, o dicho de otra forma, en este tipo de investigación se "toma prestadas" las experiencias y reflexiones de las personas para poder comprender el significado profundo de aspectos de la experiencia humana, o como dice Van Manen (2003, p. 80), "[...] reunimos las experiencia de otras personas porque nos permite ser más experimentados". Para llevar a cabo este tipo de investigaciones es necesario combinar distintos instrumentos de recogida de información, ya que existen ventajas al combinar diferentes estrategias, dado que la información obtenida en cada una de esas opciones puede ser utilizada para 
iluminar otras informaciones, o bien, ayudar a producir informaciones distintas, y por tanto, la posibilidad de diferentes conclusiones (VAN MANEN, 2003). Es por ello, que en esta investigación hemos utilizado diferentes instrumentos de recogida de datos: observación participante, grupos de discusión, diario de campo, entrevistas individuales, entrevistas en profundidad y documentos del alumnado (autobiografías).

\section{Aportaciones finales}

Somos conscientes que dar la voz al alumnado nos ha proporcionado una mirada a la que no estamos acostumbrados, porque enfoca aspectos centrales de la formación desde las posiciones que habitualmente ocupa el alumnado. Escucharles en su formación inicial nos ha permitido conocer a fondo lo que significa la experiencia de sus estudios universitarios y poder analizarla desde la perspectiva de los estudiantes. De esta manera, intento aportar algunas reflexiones desde los intereses iníciales de este trabajo, es decir, la formación inicial del alumnado de tercero de magisterio en educación física en la Universidad de Almería en base a la pregunta: ¿Qué siente, piensa, dice y hace el alumnado de magisterio de educación física en su formación inicial?

Buscar los significados que el alumnado da a sus estudios desde esta cuestión, ha supuesto el desafío de darnos cuenta que no hay final explícito ni conclusiones acabadas, máxime cuando moverse cualitativamente por la comprensión del mundo a través de la mirada del alumnado es una realidad educativa, social e institucional compleja. Conscientes de que este tipo de trabajo nunca se termina y sabiendo las limitaciones humanas que conlleva explorar la realidad desde la investigación, sí es importante dar final a un trabajo que invita a continuar comprendiendo la formación inicial del alumnado, en estos $\mathrm{y}$ otros aspectos, pero se hace necesario poner un punto y final. El punto y aparte será cuestión de futuras investigaciones. 


\subsection{EleGiR ESTUdIOS... DECISIONES, ILUSIONES... Y TAMBIÉN DESILUSIONES}

Los datos de esta investigación han puesto de manifiesto que no es fácil para el alumnado decidir qué carrera universitaria elegir, sobre todo, cuando hay situaciones familiares, presiones sociales y contextos culturales que obligan al alumnado a hacer lo que se espera que tiene que hacer y dónde. Hemos encontrado mucha vulnerabilidad emocional en el momento de la elección de estudios universitarios, ya que es una decisión importante en la vida del alumno/a. Equivocarse en la elección de la carrera genera frustración, desmotivación, baja autoestima, sentimiento de dudas, inseguridades, ganas de abandonar, ya que muchas veces, no encuentran significados al sentido de su formación. Tomar un camino equivocado significa volver atrás y comenzar de nuevo, abandonar, y en algunos casos, reconocer la equivocación, asumir el error y descubrir que hay otros caminos a seguir, otros puentes que cruzar, empezar en otra carrera. Algunos/ as, como hemos visto, aprovechan magisterio para conseguir entrar en otras titulaciones. En estas situaciones, una parte del alumnado desarrolla mecanismos de compensación para conseguir el éxito, buscando carreras de nota de corte bajo, o carreras que imaginan que van a necesitar poco desgaste para superar con éxito los estudios, como es el caso de la especialidad de magisterio de educación física en relación a la licenciatura de ciencias de la actividad física y el deporte. Otra parte del alumnado, como manifiestan las informaciones obtenidas, usa esta titulación como cajón de sastre. Y otra, ante el temor de no seguir estudios universitarios, mejor el remedio que la enfermedad. Si para acceder a los estudios basta la nota mínima, en comparación con la que se exige para cursar otros, si los que acceden a la carrera lo hacen en segunda, tercera o cuarta opción, nos encontramos con unos estudios devaluados académica y profesionalmente. 
5.2. LA FRIALDAD DE LA ACOGIDA, PRIMEROS PASOS QUE ANUNCIAN UNA FORMACIÓN DE SUPERVIVENCIA

Reiteradamente el alumnado expresa no sentirse acogido, encontrarse perdido, no saber moverse con autonomía en la universidad, tener dificultad para gestionar trámites, dada la cantidad y variabilidad de informaciones que hay que manejar en los inicios; no conocer los distintos espacios, tiempos, obligaciones y deberes, así como, las oportunidades que puede brindar la institución. Todo esto les hace vivir "lo universitario" y la Universidad como algo extraño, lejano, inabarcable y en algunos casos sufrible. Las informaciones obtenidas nos ha permitido ver, por un lado, que esta forma de acoger y sentirse acogido determina la actitud con la que el alumnado acoge su formación; y de otro, que acoger no es sólo una cuestión de recibimiento inicial, sino también de cómo la formación acoge otros modos de enseñar que apunten hacia lo que el alumnado nos ha manifestado como carencias: trabajo en equipo, reflexivo, colaborativo y dialógico, que incorpore además de lo intelectual la dimensión emocional y social. El alumnado echa en falta caminar junto a, compartir experiencias, vivencias, dificultades, sentimientos y sensaciones... que surgen a lo largo de su formación.

\subsection{Del MOdELO TRADICIONAL DE ENSEÑANZA A LA DECLARACIÓN DE BOLONIA... LA UNIVERSIDAD QUE ENSEÑA Y APRENDE}

La Universidad, desde la mirada del Espacio Europeo se convierte en oportunidad para hacer de la formación superior un espacio de aprendizajes innovadores, creativos, reflexivos... que ejerzan de puente entre el "saber y el hacer" y vincule capacidad y competencia, para hacer frente a los retos de una sociedad globalizada. Se hace necesario replantear principios, propósitos, medios y fines de la formación universitaria y ello requiere reinventarse como docentes y como aprendices, reconsiderando el rol del profesorado y del alumnado. Nuevas responsabilidades surgen en esta nueva concepción universitaria, a saber, ejercer la creatividad 
en el aprendizaje como forma para desarrollar capacidades: aprender leyendo, investigando, experimentando, interactuando con el medio, resolviendo problemas, integrando conocimiento adquirido $\mathrm{y}$ aprendizajes anteriores...

Sin embargo, las aportaciones del alumnado muestran, de forma reiterada, que la mayor parte de lo que aprenden en la universidad son conocimientos enciclopédicos, memorísticos, donde la repetición y reproducción de rutinas y recetas son la base de su aprendizaje. Destacan que muchos de sus aprendizajes son mecánicos, que los contenidos teóricos y prácticos son reiterativos y se solapan, incluso en algunas ocasiones, se ven desde distintas perspectivas contrapuestas en diferentes asignaturas. Insisten en decir que su formación sigue un modelo tradicional, centrado en la figura del profesorado, donde el alumnado es mero receptor de información. La transmisión de conocimiento viene por parte del profesorado y es él quien determina los criterios de éxito o de fracaso del proceso de enseñanza. Éste organiza y elabora los contenidos a transmitir y pauta la forma de adquirir el conocimiento. Comentan que las rutinas diarias en las aulas están organizadas, ordenadas y programadas de la misma forma, unidireccionalmente y con un único objetivo, transmitir contenidos propios de las materias. Expresan vivir la enseñanza de forma despersonalizada, masificada y unificada.

Y todo ello unido a un modelo en el que el alumnado asume un papel pasivo, con ausencia en la toma de decisiones, a la espera de lo que dicte el profesorado, con dificultades para cuestionar y vincular teoría y práctica, en el que la mayor preocupación es estudiar y entregar trabajos a tiempo para aprobar, y en algunos casos, recurrir a estrategias de engaño. El alumnado expresa con insistencia, que los sentimientos más comunes durante su formación son agobio, cansancio, saturación y desmotivación.

\subsection{EL MIEDO... UNA EMOCIÓN PRESENTE}

El miedo, la vergüenza, la inseguridad y el temor, son las emociones más claras que forman parte del bagaje experiencial que acompaña al alumnado, desde los primeros tiempos de escolarización 
hasta la formación universitaria, tal y como nos lo cuentan. El miedo incorporado a sus cuerpos inhibe la participación. Sienten vergüenza, temor y ridículo a expresar lo que no saben o creen no saber, y aún más, si se trata de compartir lo que piensan y sienten. De ahí, que preguntarles en este trabajo acerca de lo que piensan, sienten y hacen en su formación, nos haya mostrado que el alumnado no se atreve a formular preguntas por el miedo a ser "señalado". Las preguntas son importantes porque surgen en momentos de dudas, en momentos de reconocimiento o de readecuación del discurso por parte del profesorado. De otra parte, el alumnado manifiesta vivir con temor la pregunta del profesorado, por miedo a la no adecuación y a no comprender que errar es una forma de aprender. Esto significa que el profesorado ha de entender que el error es una posibilidad de aprendizaje. Si el alumnado no vive la experiencia de preguntar y errar, ¿cómo y dónde aprenderá a formular preguntas que les permita perder el miedo al error, la seguridad en la formulación de sus dudas y la confianza para expresarse entre iguales?

\subsection{EL DESENCUENTRO ACADÉMICO... LA OPORTUNIDAD PERDIDA PARA EL ENCUENTRO HUMANO Y PUENTE PARA ENCONTRAR "LO FORMATIVO"}

El alumnado ha manifestado que en su formación existen discursos autoritarios, distantes, que no son escuchados, que tienen excesivas tareas a ejecutar, que el profesorado permanece en su "tarima de marfil" y que las distancias son inaccesibles. Ante esta realidad expresada, el desencuentro académico es evidente.

Uno de los elementos que más destacan como desencuentro es lo relativo a la forma de evaluar y calificar. Hay que destacar que el alumnado no tiene clara la diferencia entre estos dos conceptos y actuaciones, y que tanto una como otra, piensan que se dan en simultaneidad. De otra parte, dicen que el profesorado es a lo que más importancia da, ya que la usan para supeditar el proceso de aprendizaje a los retos a conseguir; retos que se simplifican en la obtención de calificaciones. La forma de evaluación es la que determina el aprendizaje del alumnado, buscando en ocasiones, como dice Doyle, aprendizajes de compensación para el éxito, o sea, con 
poco aprendido obtener máxima nota. También destacan que esas actuaciones vienen de la mano de compensaciones extra curriculares. Este forma de vinculares al proceso de enseñanza aprendizaje crea una distancia que enfrenta intereses académicos y personales, e incluso a veces, profesionales. Por ello, la evaluación, lejos de ser un espacio para comprender como se ha ido realizando el proceso de aprendizaje, es un fin que aumenta el desencuentro en la relación docente.

En cuanto a la forma de realizar tareas académicas, con frecuencia basadas en la realización de trabajos en grupo, el alumnado plantea, no tanto un desencuentro con el profesorado sino un desencuentro entre iguales. Sin embargo, el planteamiento didáctico de estas tareas está, una vez más, contextualizado en la dinámica que se vive día a día en la formación universitaria (prisas, agobios, multiplicidad de "trabajitos"...), reflejando un modelo de formación inicial que focaliza su aprendizaje en la suma de partes y no en la profundización de las mismas. El alumnado expresa con insistencia que este herramienta, lejos de favorecer la integración de distintos puntos de vista, abunda en el desarrollo de estrategias engañosas (copiar de internet, trabajos de otros cursos, encubrir procesos de participación...), y ponen el énfasis en la tarea como resultado y no como proceso, aumentando así los desencuentros entre iguales.

Por eso valoran experiencias en las que el espacio del aula se convierte en lugares de escucha, de silencios, de preguntas, de experiencias que hablen de sus vidas, de cuerpos que sienten... Es importante que exista un "estar" con el otro para favorecer el encuentro humano, y así, dar "otro" sentido a su formación. Aprender a escuchar al otro está relacionado con el aprendizaje del diálogo. Sin escucha no existe diálogo. El diálogo requiere intercambios, espacio interno, curiosidad amorosa y disponibilidad para el otro. Estos son aprendizajes necesarios para quién desea dedicarse a la docencia y trabajar con personas.

5.6. Disfrutar, jugar... conceptos lúdico-motrices, ambivalencia en el aprendizaje 
Es común encontrar en las aportaciones del alumnado valoraciones como: "lo que más nos gusta es la parte práctica, ir al pabellón, tener prácticas en la naturaleza, en la piscina, en la sala de psicomotricidad...; cuando estamos en las prácticas de los coles, lo mejor de todo, es cuando estamos en el patio, o cuando nos dejan hacerles sesiones prácticas...; porque de lo que se trata es de disfrutar y que los niños y niñas también disfruten con las cosas que hacemos...; no hay nada mejor que ver a un niño o niña disfrutar y que se lo pase bien...; en el prácticum nos damos cuenta de lo poco que se juega en los coles y se sabe que jugar es muy importante para aprender, es como realmente se disfruta...; en la universidad nos dicen lo importante del juego para aprender, sin embargo, en la formación disfrutamos poco...". Disfrutar se opone a aprender y aprender no está ligado a disfrutar. Según dice el alumnado, la formación que reciben en las clases de la universidad es demasiado teórica, centrada en trasmitir conceptos y conocimientos intelectuales. El aprendizaje se aleja del disfrute. Si el aprendizaje acontece en escenarios reales, como el prácticum, es probable que se disfrute, pero desvinculado del conocimiento teórico. El alumnado expresa con frecuencia que teoría y práctica, en la formación, no va de la mano. La idiosincrasia de "lo motriz", incorpora el juego como medio de trasmisión, como instrumento básico para el aprendizaje de habilidades motrices y corporales y el "placer" como la expresión propia del movimiento. Cuando juego, placer y movimiento se unen, el disfrute emerge como experiencia vivida. El alumnado en sus aportaciones, destaca disfrutar como lo más importante para aprender, pero no cuestiona ni vincula los aprendizajes que encierra disfrutar. Para ellos y ellas, disfrutar es sinónimo de pasarlo bien, de ocuparse poco, de pasar el rato, de divertirse, de no pensar en nada ni nadie, de estar un rato dejando los problemas a un lado, de desconectar de los agobios, obligaciones y estrés que suponen las clases, el estudio, los trabajos... Argumento perfecto para atribuir al deporte, al juego, a la actividad lúdica y motriz, como placebos que separan y distancian cada vez más, lo intelectual de lo motriz, lo cognitivo de lo sensorial, el pensamiento de la expresión, lo aprendido académicamente con lo que se enseña corporalmente. 
El proceso de investigación llevado a cabo, descubre que en la formación del alumnado faltan planteamientos, propuestas y dinámicas donde se vincule "placer" y trabajo; dónde involucrase en los procesos signifique cambiar, construir, dudar y superar; donde aprender sea un ejercicio de "enamoramiento" de la realidad educativa y de las personas que la habitan; y dónde disfrutar lleve incorporado exigencia, paciencia, reflexión, dedicación, persistencia y constancia. Aprender desde lo lúdico también significa "jugar" desde la participación, el contacto, el conflicto; el "juego" de adentrarse en los pensamientos y sentimientos del alumnado. Porque enseñar y aprender es un "juego lúdico", dónde no existe adversario ya que no hay nadie a quién derrotar ni vencer; donde errar es comenzar, buscar, proponer y arriesgar...

\subsection{LA EXPERIENCIA CORPORAL... MÁS ALLÁ DE LO MOTRIZ}

La experiencia de un cuerpo holístico ha sido el gran ausente en los discursos del alumnado. En las reflexiones que aportan, podemos apreciar la ausencia de un cuerpo vivencial, expresivo, comunicativo... ("cuerpo sentido", "cuerpo con presencia") que aporte significados acerca de la variabilidad y multiplicidad que implica lo corporal. Hacen cosas con sus cuerpos, poseen esquemas cognitivos que fomentan sobre todo la automatización y la repetición. Desarrollan prácticas motrices estereotipadas, pensando y aspirando al movimiento enérgico, técnico y eficiente. Incorporar al análisis de lo gestual, lo experimental, la capacidad para expresarse con una gama amplia de movimientos y experiencias es un reto pendiente. Cuando el alumnado, al final de su formación, relata en sus intervenciones algunas de las experiencias en este sentido, descubre o re-descubre su cuerpo desde una dimensión comunicativa y expresiva. Nos aportan que haber cursado asignaturas que les plantean otra forma de acceder al conocimiento, en este caso corporal, de una forma distinta a la que tradicionalmente han tenido, les hace darse cuenta de un conocimiento que ya tenían, que recuperan y que le ayuda a dar sentido a sus cuerpos, a su elección y a su formación. 
Por ello y ante las informaciones que el alumnado nos ha proporcionado acerca de los contenidos y metodologías aprendidos en su formación en materia específica de la educación física y centradas en la técnica, el aprendizaje motor, la teoría del entrenamiento y el desarrollo de las capacidades físico-motrices, es importante y necesario ofrecer y ampliar las posibilidades formativas del alumnado. Han valorado la importancia de tener experiencias vivenciales y conscientes de movimiento dentro de una concepción integral del cuerpo, dónde la educación física pueda centrarse en el sujeto que aprende, se relaciona, se comunica y se expresa a partir de su corporalidad. Desde esta perspectiva, educar el status de la corporalidad de cada discente no consiste sólo en incluir nuevas prácticas deportivas, de juegos e incluso de condición física, sino abrir distintas posibilidades de experiencias corporales, superando la mecanización de movimientos estereotipados, estimulando la creatividad, la expresión, la sensibilidad y la conciencia corporal. De igual forma, lo apuntado por el alumnado, sugiere que las propuestas donde han tenido que pensar acerca de lo que hacían con sus cuerpos a través del movimiento, porqué, cómo y para qué lo hacían, ha sido una de las oportunidades para cuestionarse y comprender un concepto clave y nuevo en su formación, la corporeidad.

\section{Amodo de cierRe...}

Este estudio no pretende concluir, y sí ser recreado con nuevos análisis e investigaciones que amplíen aspectos inconclusos o apenas esbozados. Consideramos que hay poco estudios donde la voz del alumnado sea el hilo conductor de un proceso de investigación. De ahí la importancia y la relevancia para considerar que las voces de todos los actores de los escenarios de investigación deben estar presentes, si queremos acercarnos a la comprensión de la realidad educativa.

Parafraseando a Fedora Aberastury (2005), "las palabras (voces) son acciones", nos lleva a proponer para futuro, indagar en la conciencia que aporta ser conscientes de lo que se dice y cómo se 
dice y de cómo ello crea y genera conciencia corporal. En el terreno de la motricidad, impregnado, la mayor parte de las veces, por la mecanicidad y el tecnicismo, se abre un campo importante de indagación e investigación, en la medida que vayamos considerando que lo corporal también habla de expresiones y emociones, y que ello constituye modos de construir la corporeidad desde puntos de vista multidisciplinares.

Para esto, es necesario superar propuestas pedagógicas que conducen a la acomodación y la repetición, dónde el alumnado deje de ser pasivo, redireccionando la mirada a una pedagogía que se fundamente en la flexibilidad, creatividad y diálogo. Buscar marcos epistemológicos más amplios y humanísticos, que permitan repensar prácticas pedagógicas más acordes a los sujetos actuales. Sería importante conducir la formación hacia la posibilidad de vivir la complejidad, la incertidumbre y la tensión de la vida real y de la comunidad educativa, arropados por una efectiva y activa cooperación entre iguales y tutorizados por profesionales expertos que acompañen y orienten la formación desde los primeros momentos que el alumnado ingrese en la universidad.

Futuras investigaciones pueden hacer propuestas para abordar experiencias reales que se están llevando a la práctica y que muestran una dimensión integradora y globalizadora del hecho educativo. Dar la voz a los y las participantes de estas experiencias podría enriquecer, orientar y sugerir los beneficios, mejoras y retos a tener en cuenta para una formación inicial acorde con las propuestas de una pedagogía crítica o de una "pedagogía para la esperanza" en palabras de Freire. En este mismo sentido se hará necesario investigaciones que revisen las teorías pedagógicas que subsisten en el interior de la formación inicial de los docentes de educación física, que impiden comprender o abordar nuevas expresiones, vivencias y experiencias de movimiento que atienda al sujeto integralmente, donde el alumnado pueda vivir una experiencia de transformación, superando una formación técnica, mecánica, fragmentada y estereotipada.

Por último, sugerimos el desarrollo de líneas de investigación acerca de la formación de futuros profesores y profesoras, que 
aborde experiencias de cómo la voz del estudiante construye distintos mundos de identidades y subjetividades dando sentido a los diferentes modelos corporales que cohabitan en el aula y de cómo lo corporal significa modos y maneras de ejercer lo motriz, lo emocional, lo cultural. 
The voice of students: a narrative investigation
about what the physical education students
feel, think, and do in their initial training.
Abstract: This research aims to describe and interpret
what the student of physical education whose area
of study is teaching feels, thinks, says and does in
his/her initial training. Different instruments were used
to collect information: participant observation, panel
groups, individual interviews, in-depth interviews and
the student's autobiographies. I propose at the end of
the paper that we continue investigating in this area
the possibility of living the complexity, uncertainty and
tension of real life in educational settings and in this
way broaden the reflection of initial training.
Keywords: Students. Education. Cultural anthropology.

A voz dos alunos (as): Uma investigação narrativa sobre o que sente, pensa, diz e faz o alunado de Educação Física em sua formação inicial.

Resumo: Esta investigação descreve e a interpreta o que sente, pensa, diz e faz o aluno (a) de magistério especialidade educação física em sua formação inicial. Foram utilizados diferentes instrumentos de coleta de informações: observação participante, grupos de discussão, entrevistas individuais, entrevistas em profundidade e documentos dos alunos. Proponho que se continue investigando com a possibilidade de viver a complexidade, a incerteza e a tensão da vida real em contextos educativos e que se amplie a reflexão da formação inicial.

Palavras- chave: estudantes. Educação. Antropologia cultural

\section{REFERENCIAS}

ABERASTURY, Fedora. Sistema consciente para la técnica del movimiento. Buenos Aires: Catálogos Editora, 2005.

DELORS, Jaques. La educación encierra un tesoro: Informe de la Comisión Internacional sobre la educación para el siglo XXI. Santilla: UNESCO,1996.

EISNER, Elliot. El Ojo llustrado-Indagación Cualitativa y Mejora de la Práctica Educativa. Barcelona: Paidós, 1998.

ERICKSON, Frederick. Métodos cualitativos de investigación sobre la enseñanza. En: WITROCK, M.C.. (Ed.), La investigación de la enseñanza II: Métodos cualitativos y de observación. Barcelona: Paidós, 1989. P. 248. 
FERNÁNDEZ-BALBOA, Juan. Miguel. La sociedad, la escuela y la educación física del futuro. En: DEVÍS,J.; FERNÁNDEZ-BALBOA, J. M.; MEEK, G.; JIMENO, L. A.; EVANS, J. ; SPARKES, A. La educación física, el deporte y la salud en el siglo XXI. Madrid: Editorial Marfil, 2001. P. 356.

FLICK, Uwe. Introducción a la Investigación Cualitativa. Madrid: Morata,2004.

FREIRE, Paulo. La educación como práctica de la libertad. Madrid: Siglo veintiuno de España, 2002.

GIMENO, José; PÉREZ GÓMEZ, Angel. Comprender y transformar la enseñanza. Madrid: Morata, 2005.

HAMMERSLEY, Martyn; ATKINSON, Paul. Etnografía Métodos de investigación. Barcelona: Paidós,2001.

KUSHNER, Saville. Personalizar la evaluación. Madrid: Morata, 2002.

RIVAS, José Ignácio. Investigación Naturalista en Educación: una Revisión Crítica.Valencia: Promolibro, 1990.

TAYLOR, Steven; BOGDAN, Robert. Introducción a los métodos cualitativos de investigación. La búsqueda de significados. Barcelona: Paidós, 2002.

VAN MANEN, Max. Investigación Educativa y experiencia vivida. Barcelona: Idea Books, 2003.

WOLCOTT, Harry. Sobre la intención Etnográfica. En: Rada, A.; Velasco, H..; García, F..; Malinowski, b.; Wolcott, H.; Hymes, D.. Lecturas de antropología para Educadores: el ámbito de la antropología de la educación y de la etnografía escolar. Madrid: Trotta, 1993. p. 127-144.

Endereço para correspondência:

Berenice Mattos Medina

Rua XV de Novembro, 1010 apt. 303

Pelotas-RS

Cep: 96015-000

Recebido em: 13.03.2013

Aprovado em: 09.06.2013 